\title{
Effect of low level substitution of Sr-Ba on transport and magnetic behaviour of $\mathrm{La}_{0.67} \mathrm{Ca}_{0.33} \mathrm{MnO}_{3}$
}

\author{
B MUNIRATHINAM, M KRISHNAIAH* ${ }^{*}$ M MANIVEL RAJA ${ }^{\dagger}$, S ARUMUGAM ${ }^{\dagger \dagger}$ \\ and K PORSEZIAN" \\ Department of Physics, Sri Venkateswara University, Tirupati 517 502, India \\ ${ }^{\dagger}$ Advanced Magnetics Group, Defence Metallurgical Research Laboratory, Hyderabad 500 058, India \\ $\dagger^{\dagger}$ Department of Physics, Bharathidasan University, Tiruchirapalli 620 024, India \\ \#Department of Physics, Pondicherry University, Puducherry 605 014, India
}

MS received 25 June 2010; revised 5 September 2010

\begin{abstract}
In this paper we report the investigation of transition metal oxide compound, $\mathrm{La}_{0.67} \mathrm{Ca}_{0.25} \mathrm{Sr}_{0.04} \mathrm{Ba}_{0.04} \mathrm{MnO}_{3}$ (LCSBMO), along with $\mathrm{La}_{0.67} \mathrm{Ca}_{0.33} \mathrm{MnO}_{3}$ (LCMO), synthesized by sol-gel route under identical conditions. The effect of simultaneous low level substitution of large size ions such as $\mathrm{Sr}^{2+}$ and $\mathrm{Ba}^{2+}$ for $\mathrm{Ca}^{2+}$ ions on the electronic transport and magnetic susceptibility properties are analysed and compared apart from microstructure and lattice parameters. The temperature dependent electrical transport of the polycrystalline pellets of LCSBMO and LCMO when obeying the well studied law, $\rho=\rho_{0}+\rho_{2} T^{2}$ for $T<T_{M I}$, is observed to differ by more than $50 \%$ from the values of $\rho_{0}$ and $\rho_{2}$, with the former compound showing enhanced electrical conductivity than the latter. Similarly in fitting the adiabatic small polaron model for resistivity data of both the samples for $T>T_{\text {MI }}$, the polaron activation energy is found to differ by about $11 \%$. In addition, the temperature dependent a.c. magnetic susceptibility study of the compounds shows a shift of about $6 \%$ in the paramagnetic to ferromagnetic transition temperature (285 K for LCSBMO and $270 \mathrm{~K}$ for LCMO).
\end{abstract}

Keywords. Ceramic; sol-gel processes; magnetic measurements; electronic transport; X-ray diffraction.

\section{Introduction}

The optimally doped composition $(x=0 \cdot 33)$ of the intermediate bandwidth manganite system, $\mathrm{La}_{1-x} \mathrm{Ca}_{x} \mathrm{MnO}_{3}$, has been studied extensively since the observation of large negative magneto resistance and strong ferromagnetism (Jin et al 1994). In this compound, the double-exchange mechanism (DE) and electron-lattice coupling are believed to play major role in the occurrence of phenomena such as ferromagnetism (FM), insulator-metal transition and colossal magneto resistance (Millis et al 1995). It is known that the substitution of cations of different sizes for $A$-site ions $\left(\mathrm{La}^{3+}\right.$ and $\left.\mathrm{Ca}^{2+}\right)$ affects the unit cell structure and the corresponding changes in the distortion or rotation of $\mathrm{MnO}_{6}$ octahedrons give rise to a wide variety of electronic properties (Hwang et al 1995). The conduction bandwidth of $e_{\mathrm{g}}$ electrons of $\mathrm{Mn}$ ions for instance is known to be strongly affected by the average size of $A$-site cations. By increasing the value of the latter by substitution method, both the conductivity and the paramagnetic to ferromagnetic transition temperature $\left(T_{\mathrm{C}}\right)$ may be increased. In this context, the electronic transport and the magnetic susceptibility properties of optimally doped compounds, $\mathrm{La}_{0.67} \mathrm{Ca}_{0.25} \mathrm{Sr}_{0.04} \mathrm{Ba}_{0.04} \mathrm{MnO}_{3}$

\footnotetext{
*Author for correspondence (bmuniratnamisro@gmail.com)
}

(LCSBMO) and $\mathrm{La}_{0.67} \mathrm{Ca}_{0.33} \mathrm{MnO}_{3}$ (LCMO), synthesized under identical conditions are studied and compared. In the former composition, the average size of $A$-site ions has been increased lightly by simultaneous substitution of small percentage of large size ions such as $\mathrm{Sr}^{2+}$ and $\mathrm{Ba}^{2+}$ for $\mathrm{Ca}^{2+}$ ions. The focus of this work has been to study the effect of this low level substitution on the structural, microstructure, electronic transport and magnetic susceptibility properties of this widely studied LCMO. The electrical parameters which arise out of the fitting of standard models with resistivity data for $T<T_{\mathrm{MI}}$ and $T>T_{\mathrm{MI}}$ are compared and discussed in the above context apart from the paramagnetic to ferromagnetic transition temperatures $\left(T_{\mathrm{C}}\right)$.

\section{Experimental}

The polycrystalline sample, LCSBMO, was synthesized along with LCMO through the low temperature sol-gel route. $99.9 \%$ Pure chemicals of $\mathrm{La}\left(\mathrm{NO}_{3}\right)_{3} \cdot 6 \mathrm{H}_{2} \mathrm{O}, \mathrm{Ca}\left(\mathrm{NO}_{3}\right)_{2} \cdot 4 \mathrm{H}_{2} \mathrm{O}, \mathrm{Ba}\left(\mathrm{NO}_{3}\right)_{2}, \quad \mathrm{Sr}\left(\mathrm{NO}_{3}\right)_{2}$ and $\mathrm{Mn}\left(\mathrm{CH}_{3} \mathrm{COO}\right)_{2} \cdot 4 \mathrm{H}_{2} \mathrm{O}$ were used to prepare individually the solutions of specific molarity using distilled water. The metal solutions were then mixed together in specific amounts to ensure desired stoichiometric amount of metallic cations. The mixed solutions were thoroughly stirred using magnetic 
stirrers and then citric acid was added in a specific molar ratio with respect to cations. The $\mathrm{pH}$ of each solution was adjusted to neutral value using the aqueous $\mathrm{NH}_{3}$ solution to avoid precipitation. The solutions were then slowly evaporated at $95^{\circ} \mathrm{C}$ on a hot plate to get gel. The gels were further dried at $300^{\circ} \mathrm{C}$ until each self-ignited and burnt into soft black powder. To obtain well crystallized single-phase oxide, the above black powders were calcined in air for $7 \mathrm{~h}$ at $900^{\circ} \mathrm{C}$ and furnace cooled to ambient temperature $\left(25^{\circ} \mathrm{C}\right)$ gradually. The powder was then ground thoroughly in a mortar and again calcined at $900^{\circ} \mathrm{C}$ for another $7 \mathrm{~h}$ before furnace cooling gradually to ambient temperature. Around $1 \mathrm{~g}$ of each of the powder samples was then pressed into pellets and sintered in air at $900^{\circ} \mathrm{C}$ for $14 \mathrm{~h}$. The XRD patterns of LCMO and LCSBMO were recorded with $\mathrm{Cu}-\mathrm{K}_{\alpha}$ radiation. The electrical resistance of pellets as a function of temperature was measured by the conventional d.c. four-probe technique using a closed cycle He-cryostat. A programmable current source was used to apply constant current and the resulting voltage was measured with a programmable voltmeter. The temperature dependent a.c. magnetic susceptibility measurement of the samples was carried out using a.c. susceptometer with a magnetic field of strength, $5 \mathrm{Oe}$ and frequency, $430 \mathrm{~Hz}$ in the temperature range $77-340 \mathrm{~K}$.

\section{Results and discussion}

The X-ray diffraction analysis of the compounds confirms single phase formation and the corresponding patterns could be indexed to orthorhombic structures (Pbnm) using XRDA software. The lattice parameters are given in table 1 . The unit cell volume for LCSBMO is found to be marginally less than that of LCMO. The morphology of the samples

Table 1. Lattice, electronic transport and magnetic parameters of LCSBMO and LCMO.

\begin{tabular}{lcc}
\hline Lattice and transport parameters & LCMO & LCSBMO \\
\hline$a(\AA)$ & $5 \cdot 487$ & $5 \cdot 472$ \\
$b(\AA)$ & $5 \cdot 468$ & $5 \cdot 476$ \\
$c(\AA)$ & $7 \cdot 755$ & $7 \cdot 756$ \\
$V\left(\AA^{3}\right)$ & $232 \cdot 7$ & $232 \cdot 4$ \\
$<r_{\mathrm{A}}>(\AA)$ & $1 \cdot 20$ & $1 \cdot 23$ \\
$T_{\mathrm{MI}}(\mathrm{K})$ & 195 & 239 \\
$T_{\mathrm{C}}(\mathrm{K})$ & 270 & 285 \\
$\rho_{0}(\Omega \mathrm{m}) \times 10^{-3}$ & $12 \cdot 8$ & $5 \cdot 30$ \\
$\rho_{2}\left(\Omega \mathrm{m} / \mathrm{K}^{2}\right) \times 10^{-7}$ & $4 \cdot 58$ & $2 \cdot 25$ \\
$E_{\mathrm{p}}(\mathrm{eV})$ & $0 \cdot 09$ & $0 \cdot 08$ \\
$A(\Omega \mathrm{m} / \mathrm{K}) \times 10^{-6}$ & $1 \cdot 29$ & 0.97 \\
$T_{0}(\mathrm{~K}) \times 10^{6}$ & $5 \cdot 17$ & $1 \cdot 58$ \\
$N\left[E_{\mathrm{F}}\right]\left(\mathrm{eV} \mathrm{V}^{-1} \mathrm{~m}^{-3}\right) \times 10^{26}$ & $9 \cdot 50$ & $9 \cdot 51$ \\
$1 / \alpha(\AA)$ & $3 \cdot 55$ & $5 \cdot 26$ \\
$W(\mathrm{eV})$ & $0 \cdot 07$ & $0 \cdot 05$ \\
$R(\AA)$ & $15 \cdot 2$ & $16 \cdot 7$ \\
\hline
\end{tabular}

shows that all the crystallites are nearly of spherical shape with narrow size distribution. The average particle size measured from SEM photograph is around $150 \mathrm{~nm}$ for LCMO and $200 \mathrm{~nm}$ for LCSBMO. The results of energy dispersive $\mathrm{X}$-ray spectroscopy (EDS) show that $\mathrm{La}, \mathrm{Ca}, \mathrm{Sr}, \mathrm{Ba}$ and $\mathrm{Mn}$ are exchanged in a closely linear manner as intended.

\subsection{Electronic transport}

In order to understand different electronic transport mechanisms, the resistivity data of samples has been analysed separately in two different temperature regimes: (i) low temperature $\left(T<T_{\mathrm{MI}}\right)$ metallic phase regime $(d \rho / d T>0)$ and (ii) high temperature ( $T>T_{\mathrm{MI}}$ ) semiconducting or insulating phase regime $(d \rho / d T<0)$. The temperature dependant resistivity of each sample is studied in the temperature range 45-290 K. Broad transition is observed from insulating phase to metallic phase during cooling in both cases (figure 1). Apart from the broadness, the peak of the transitions, $T_{\mathrm{MI}}$, is found to be on the lower side for all the samples as compared to that of PM to FM transitions. The lower $T_{\mathrm{MI}}$ values may be due to several factors: (i) smaller average particle size of the samples than that measured using SEM, (ii) influence of extrinsic contributions such as the large number of grain boundaries, (iii) spin-polarized tunnelling between ferromagnetic grains through an insulating grain boundary layer and so on. The reason for the discrepancy in the particle size measured may be because of using SEM of a particle consisting more than one crystallite. The higher value of $T_{\mathrm{MI}}$ for LCSBMO than that of LCMO, however, reveals the role played by the mild doping of $\mathrm{Sr}^{2+}$ and $\mathrm{Ba}^{2+}$ ions as compared to that of LCMO. The $T_{\mathrm{MI}}$ observed for LCMO is, however, found to be higher than that reported for the composition, $\mathrm{La}_{0.67} \mathrm{Ca}_{0.33} \mathrm{MnO}_{3}$, synthesized at $900^{\circ} \mathrm{C}$ through

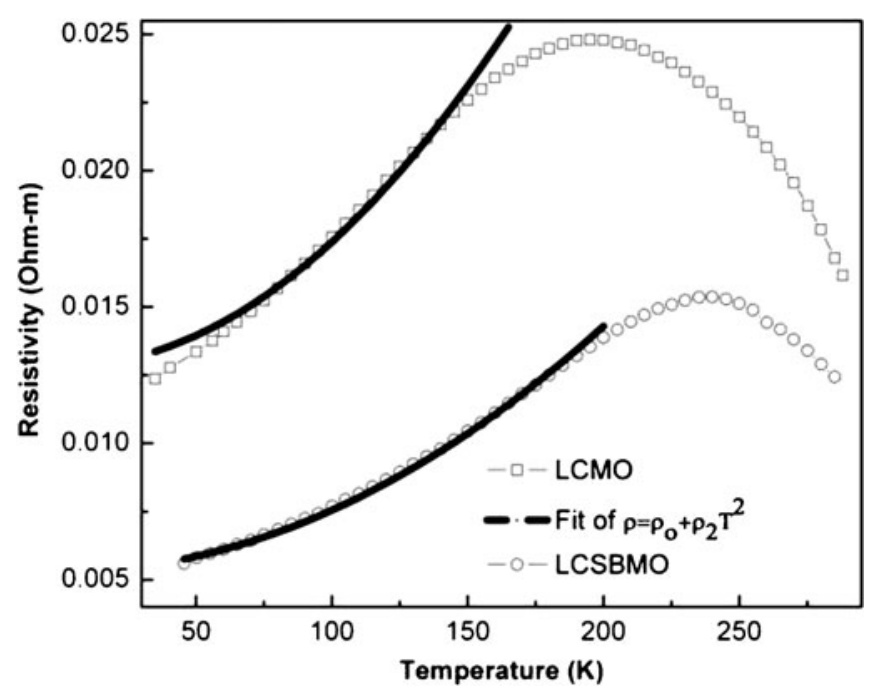

Figure 1. Temperature dependent resistivity and the fit of model $\rho=\rho_{0}+\rho_{2} T^{2}$ for $T<T_{\mathrm{MI}}$. 
sol-gel route (Venkataiah et al 2008). As $T_{\mathrm{MI}}$ has been shown to increase linearly with the mean radius of $A$-site cations in the range between $1.19 \AA$ and $1.24 \AA$ (Raveau et al 1998), the influence of size mismatch factor $\left(\sigma^{2}\right)$ appears to be negligible for the present compounds in affecting the transition temperature. The value of $\sigma^{2}$ is calculated to be $6.8 \times 10^{-5}$ for LCBSMO and $2.9 \times 10^{-6}$ for LCMO assuming 9-fold coordination for $A$-site ions.

3.1a Low temperature electronic transport $\left(T<T_{M I}\right)$ : At $T<T_{M I}$, the spontaneous alignment of Mn spins allows delocalization of $e_{\mathrm{g}}$ electrons leading to low resistivity FM phase and the resistivity data of compounds could be fitted below $T_{\mathrm{MI}}$ (correlation coefficient $(r)=0.999$ for LCS$\mathrm{BMO}$ and 0.995 for LCMO) to the following equation in the temperature range from 45-200 K for LCSBMO and from $50-150 \mathrm{~K}$ for LCMO.

$$
\rho=\rho_{0}+\rho_{2} T^{2} \text {. }
$$

Here $\rho_{0}$, representing the resistivity contribution due to temperature-independent scattering mechanisms including that of grain and domain boundary effects is found to be lower by about $59 \%$ for LCSBMO as compared to LCMO (table 1). Similar is the trend (about $51 \%$ lower for LCSBMO) for $\rho_{2}$, which measures the contribution from electron-electron scattering. In addition, the term such as $\rho_{2.5} T^{2.5}$, which represents a combination of electronelectron, electron-phonon, and electron-magnon scattering (Schiffer et al 1995) is also found to fit the data adequately $(r=0.99)$. It appears that the lower value of $\rho_{2.5}(=7.08 \times$ $10^{-9} \Omega \mathrm{m} / \mathrm{K}^{2}$ ) for LCSBMO may also be the cause for the start of spin ordering at higher temperature and hence the higher value of PM to FM transition temperature as compared to that of LCMO $\left(\rho_{2.5}=5.04 \times 10^{-8} \Omega \mathrm{m} / \mathrm{K}^{2}\right)$. In other words, the DE links appear to be pronounced resulting in an overall increase in the bandwidth of $e_{\mathrm{g}}$ electrons and the consequent enhanced ferromagnetic interaction for LCSBMO gives rise to higher $T_{\mathrm{C}}$. It is to be noted, however, that the resistivity data for LCMO could not be fitted with (1) below about $50 \mathrm{~K}$ (figure 1) indicating the need to look for unconventional scattering processes that go beyond the rigid band approximation and take into account spin fluctuations at low temperatures (Furukawa 2000).

3.1b High temperature electronic transport $\left(T>T_{M I}\right)$ : In the temperature range above $T_{\mathrm{MI}}$, the electrical resistivity of manganites generally exhibit strong temperature dependence. Among the popular resistivity, $\rho(T)$ models, adiabatic small polaron model $\left[\rho=A T \exp \left(E_{\mathrm{p}} / k_{\mathrm{B}} T\right)\right]$ having its origin from the local lattice distortion accompanying the moving charge carrier (Jahn-Teller polaron); and variable range hopping (VRH) model $\left[\rho=\rho_{\infty} \exp \left[\left(T_{0} / T\right)^{1 / 4}\right]\right]$ with the localization of charge carriers by the magnetic disorder have been found to fit closely well with the resistivity data of both the samples for temperature range starting at $270 \mathrm{~K}$

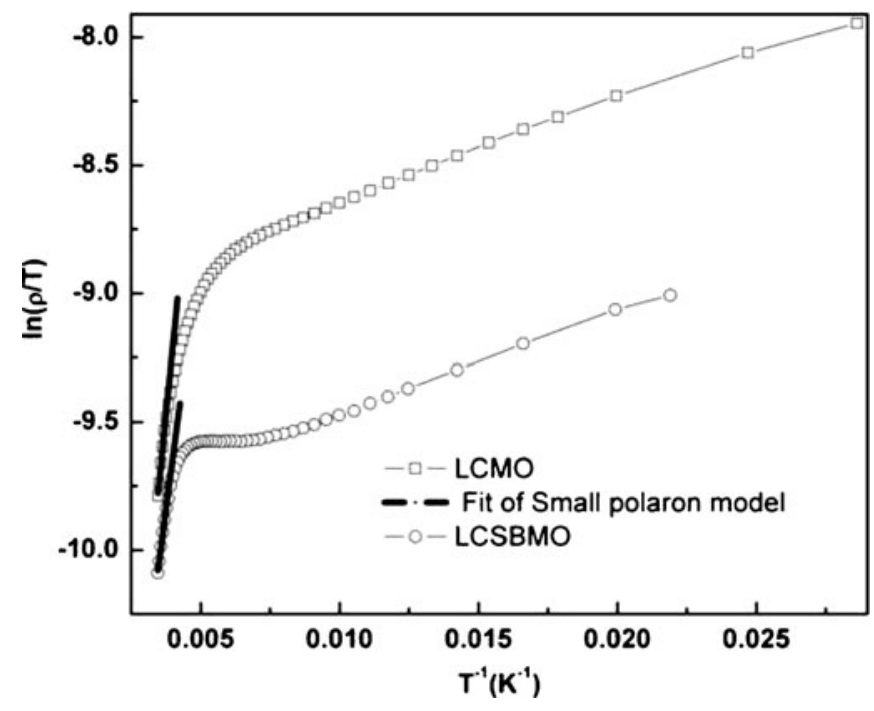

Figure 2. Fit of adiabatic small polaron model (solid curve) for $T>T_{\mathrm{MI}}$.

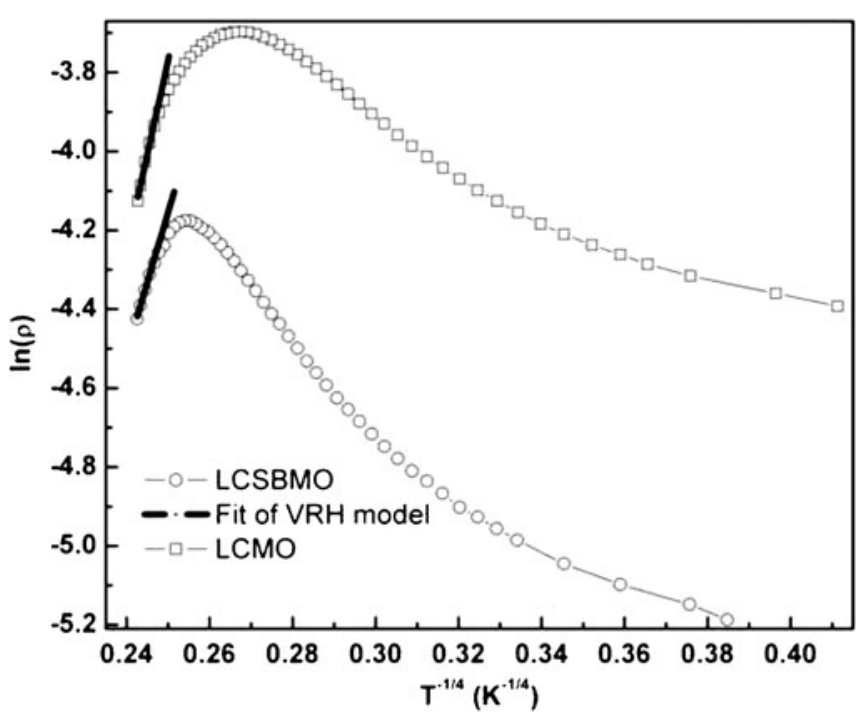

Figure 3. Fit of variable range hopping model (solid curve) for $T>T_{\mathrm{MI}}$.

(figures 2 and 3). The attempt to fit the data with the simple activation model has proved to be unsuccessful. The activation energy, $E_{\mathrm{p}}$, for nearest neighbour hopping of small polarons and temperature independent constant, $A$, have been determined from the fit of resistivity data of compounds (table 1) using the following form of polaron equation

$$
\ln (\rho / T)=\ln (A)+E_{\mathrm{p}} / k_{\mathrm{B}} T .
$$

The results show increased polaron mobility for LCSBMO as compared to LCMO, with the activation energy of the former being comparatively lower by about $11 \%$.

The resistivity behaviour for temperature $T>T_{\mathrm{MI}}$ has also been analysed within the framework of the modified VRH 
model proposed by Viret et al (1997) assuming that the carrier localization above $T_{\mathrm{C}}$ is caused by a random potential of magnetic origin. From the fit of the resistivity data, the value of characteristic temperature $\left(T_{0}\right)$ has been obtained. Using the value of $T_{0}$, the localization length $(1 / \alpha)$, the density of states, $N\left(E_{\mathrm{F}}\right)$, the carrier hopping energy, $W$ and the hopping distance, $R$, have been calculated (table 1). The lower value of $T_{0}$ for LCSBMO is reflected in the corresponding higher values of $N\left(E_{\mathrm{F}}\right),(1 / \alpha)$ and consequently $R$. The carrier hopping energy $(W)$ for LCSBMO is found to be lower by about $29 \%$ as compared to LCMO, confirming enhanced carrier mobility or the $e_{\mathrm{g}}$ electron bandwidth of the former compound.

\subsection{Magnetic susceptibility studies}

The variation of the real component $\left(\chi^{\prime}\right)$ of a.c. magnetic susceptibility with temperature is plotted in figure $4 . \chi^{\prime}(T)$ was continuously measured using a low excitation field of strength, 5Oe and a frequency of $430 \mathrm{~Hz}$ as the sample was warmed from $77-340 \mathrm{~K}$. It is to be noted that no static magnetic field was applied during the above process. The peak value of $\chi^{\prime}$ for LCSBMO is found to be about 1.3 times that of LCMO. The $\chi^{\prime}(T)$ is characterized by almost a gradual fall for both the compounds after attaining the peak as the temperature is lowered. The percentage of reduction is found to be $10 \%$ for LCSBMO and $7 \%$ for LCMO. This decreasing tendency of $\chi^{\prime}$ with temperatures particularly for ferromagnetic compositions such as these is generally attributed to the domain wall pinning effects rather than to effects such as mixed magnetic phase, re-entrant spin glass etc as similar behaviour is observed in ferromagnetic systems other than the manganites (Joy and Date 2000). The discontinuous jump exhibited in LCMO may be due to thermally and a.c.-fieldactivated domain wall jumps. The number of such jumps arising from the release of domain walls from pinning centres

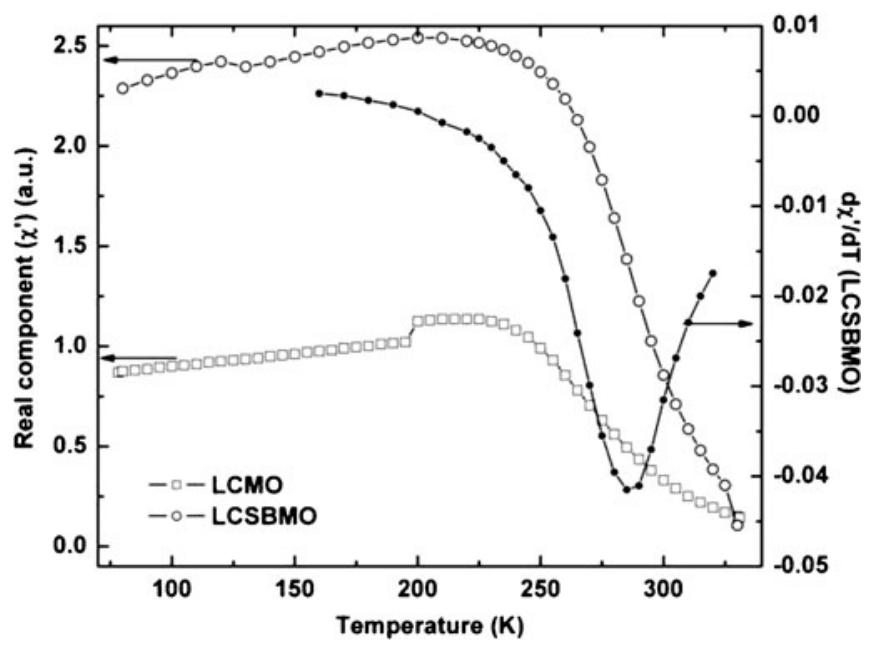

Figure 4. A.C. magnetic susceptibility vs temperature for LCSBMO and LCMO samples. as the temperature is lowered may depend on the distribution and strength of pinning centres in the respective samples (Wang et al 1998).

It has been shown that the PM-FM transition temperature $\left(T_{\mathrm{C}}\right)$ increases with the tolerance factor $(t)$ and reaches its maximum at $t_{\max } \approx 0.93$ (Hwang et al 1995). $T_{\mathrm{C}}$ shows a decreasing trend beyond this value of $t$, attributable to the effect of size mismatch of $A$-site cations. As the calculated values of $t(0.925$ for LCSBMO and 0.917 for LCMO) fall below $t_{\max }$, the effect of the mean size of the $A$-site ions clearly dominates over that of size mismatch factor in affecting the magnetic transition temperature. In this respect the differentiation of a.c. magnetic susceptibility of the sample with respect to temperature shows a higher transition temperature of $285 \mathrm{~K}$ for LCSBMO corresponding to the point of inflection (table 1) and this represents a rise of about $6 \%$ for LCSBMO as compared to LCMO. In addition, as shown by $d \chi^{\prime} / d T$ vs $T$ curve of figure 4, the magnetic transition is observed to be broad, indicative of the coexistence of both short and long range ordering of ferromagnetic clusters.

\section{Conclusions}

The mixed valence manganite, $\mathrm{La}_{0.67} \mathrm{Ca}_{0 \cdot 25} \mathrm{Sr}_{0 \cdot 04} \mathrm{Ba}_{0 \cdot 04} \mathrm{MnO}_{3}$, synthesized along with $\mathrm{La}_{0.67} \mathrm{Ca}_{0.33} \mathrm{MnO}_{3}$ through sol-gel route under identical conditions is studied for its structural, electrical and magnetic properties. The electronic transport property has been analysed using standard models and compared separately for $T<T_{\mathrm{MI}}$ and $T>T_{\mathrm{MI}}$. The electrical transport parameters calculated show enhanced electronic conductivity for LCSBMO as compared to LCMO. In other words, the polaron activation energy, $E_{\mathrm{p}}$, is seen lower by about $19 \%$ for LCSBMO as compared to LCMO. The light doping of $\mathrm{Sr}^{2+}$ and $\mathrm{Ba}^{2+}$ for $\mathrm{Ca}^{2+}$ ions has also caused about $6 \%$ shift of PM to FM transition temperature $\left(T_{\mathrm{C}}\right)$ to a higher value close to room temperature as confirmed by a.c. magnetic susceptibility measurements.

\section{References}

Furukawa N 2000 J. Phys. Soc. Jap. 691954

Hwang H Y, Cheong S W, Radaelli P G, Marezzio M and Batlogg B 1995 Phys. Rev. Lett. 75914

Jin S, Tiefel T H, McCormack M, Fastnacht R A, Ramesh R and Chen L H 1994 Science 264413

Joy P A and Date S K 2000 J. Magn. Magn. Mater. 220106

Millis A J, Littlewood P B and Shraiman B I 1995 Phys. Rev. Lett. 745144

Raveau B, Maignan A, Martin C and Hervieu M 1998 Chem. Mater. 102641

Schiffer P, Ramirez A P, Bao W and Cheong S W 1995 Phys. Rev. Lett. 753336

Venkataiah G, Lakshmi Y K and Reddy P V 2008 PMC Phys. B1 7 Viret M, Ranno L and Coey J M D 1997 Phys. Rev. B55 8067

Wang X L, Horvat J, Liu H K and Dou S X 1998 Phys. Rev. B58 2434 